\title{
Computationally Efficient, Real-Time, and Embeddable Prognostic Techniques for Power Electronics
}

\author{
Alireza Alghassi, Student Member, IEEE, Suresh Perinpanayagam, Member, IEEE, Mohammad Samie, \\ and Tarapong Sreenuch
}

\begin{abstract}
Power electronics are increasingly important in new generation vehicles as critical safety mechanical subsystems are being replaced with more electronic components. Hence, it is vital that the health of these power electronic components is monitored for safety and reliability on a platform. The aim of this paper is to develop a prognostic approach for predicting the remaining useful life of power electronic components. The developed algorithms must also be embeddable and computationally efficient to support on-board real-time decision making. Current state-of-the-art prognostic algorithms, notably those based on Markov models, are computationally intensive and not applicable to real-time embedded applications. In this paper, an isolated-gate bipolar transistor (IGBT) is used as a case study for prognostic development. The proposed approach is developed by analyzing failure mechanisms and statistics of IGBT degradation data obtained from an accelerated aging experiment. The approach explores various probability distributions for modeling discrete degradation profiles of the IGBT component. This allows the stochastic degradation model to be efficiently simulated, in this particular example $\sim 1000$ times more efficiently than Markov approaches.
\end{abstract}

Index Terms-Isolated-gate bipolar transistor (IGBT), MonteCarlo simulation (MCS), power electronics, prognostics, remaining useful life (RUL).

\section{INTRODUCTION}

$\mathbf{O}$ VER the last 20 years, electric vehicle (EV) technologies have taken a significant leap forward, primarily aided by advances in electrical motor drives, power converters, batteries, and system configurations [1]. Power electronic converters are widely used in electric traction drive applications, while multichip insulated-gate bipolar transistor (IGBT) power modules are the most commonly used power switches for EVs' on-board power converters [2]. However, IGBTs are known to be prone to failure, and the situation only worsens when operating in harsh environments, such as beneath the bonnet of an EV [3]. Table I represents typical environmental conditions and operational requirements for automotive applications. Clearly, the performance and reliability of IGBT modules are constrained by these stringent conditions, such as exposure to high

Manuscript received April 2, 2014; revised September 2, 2014; accepted September 12, 2014. Date of publication October 2, 2014; date of current version December 23, 2014. Recommended for publication by Associate Editor S. Perinpanayagam.

The authors are with the School of Applied Sciences, Integrated Vehicle Health Management Centre, Cranfield University, Cranfield MK43 0AL, U.K. (e-mail: a.alghassi@cranfield.ac.uk; suresh.nayagam@cranfield.ac.uk; m.samie@cranfield.ac.uk; t.sreenuch@cranfield.ac.uk).

Color versions of one or more of the figures in this paper are available online at http://ieeexplore.ieee.org.

Digital Object Identifier 10.1109/TPEL.2014.2360662
TABLE I

AUTOMOTIVE ENVIRONMENTAL CONDITION [3]

\begin{tabular}{lcccc}
\hline \hline Cabin & $\begin{array}{c}\text { Engine } \\
\text { Compartment }\end{array}$ & Engine & Transmission \\
\hline & & \\
\hline $\begin{array}{l}\text { Temperature Range } \\
\text { Vibration }\end{array}$ & -40 to $85^{\circ} \mathrm{C}$ & -40 to $105^{\circ} \mathrm{C}$ & -40 to $125^{\circ} \mathrm{C}$ & -40 to $150{ }^{\circ} \mathrm{C}$ \\
\hline \hline
\end{tabular}

temperatures, high levels of humidity, extreme thermal loading load cycling, and mechanical stress [3]. Among these conditions, thermomechanical stress is an overriding factor that causes IGBT packaging connector fatigue (such as bond wire and solder connection failures). Nevertheless, despite improvements in solder technologies, wire bonds are still generally observed as the major cause for packaging failures, which reduce the lifetime of IGBTs. Therefore, we have chosen wire bonds to be the focus of this paper.

At present, wire bonding is the dominant technology used to connect terminals (or leads) to semiconductor chips because it is still regarded as a straightforward, flexible, and cost-effective solution [4]. Recent advances in new composition wire materials and new bonding technologies (e.g., special coating and strain buffer bonding) have received a great deal of attention by industry. Nonetheless, when bonding using these new technologies fails, the entire device will be damaged with catastrophic consequences.

Undoubtedly, the detection of an IGBT fault prior to prognosis or even after diagnosis is of critical importance for a healthy converter operation system. Conventional vehicles are equipped with an on-board diagnostic system, which is capable of detecting a "happened" fault and flag it up to the driver of the vehicle [5], [6]. In the event of an IGBT failure, the detection must be quick enough (ideally, less than $10 \mu \mathrm{s}$ ) to prevent a fault from propagating, which places a limitation on the hardware setup [7].

Essentially, there is an increasing need for failure detection technologies to provide a state-of-health or early warning information. It is worth noting that in general, power electronic components fail quite frequently. Hence, there is no real need to have prognostics in place but if we can detect abnormality at an earlier stage, it is possible to increase the time to failure or the duration of the potential of failure (POF) (see Fig. 1). On the other hand, prognostics results can be used for optimization of system control, rather than predictive maintenance to extend the overall remaining useful life (RUL). In order to justify the 


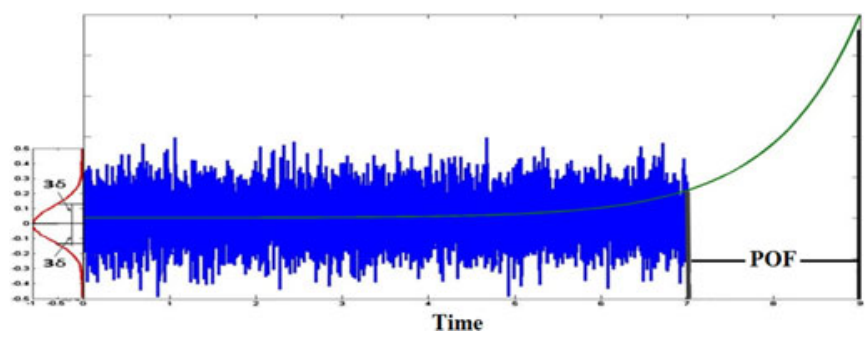

Fig. 1. Potential of Failure.

usefulness of prognostics, the duration of POF must be quite long. Therefore, it is important to know which feature must be monitored to detect malfunction at an early stage. If the monitored parameters persistently deviate by more than $3 \delta(\mathrm{Std})$ as a detection error over the flat region, this implies that there is an abnormality at this time.

Commercial reliability prediction programmes for electrical and electromechanical stress still use traditional reliability prediction methods, including Mil-HDBK-217, 217-PLUS, PRISM, Telcordia, and FIDES [6], [8]. These are empirical methods based on statistical data and average performance of a large number of identical products. In essence, these methods account little for the mechanisms of complex in-service failures. It is therefore not surprising that the results obtained from these methods do not always correlate well with actual failures in the field. Recently, some advanced prognostic techniques have been proposed to predict semiconductor devices' impending failures [9].

First, a range of detecting elements may be embedded within the host device and their failures provide an early warning signal. This is called the Canary method. The second method is the analytical lifetime prediction based on Coffin-Manson models, Norris-Landzberg models, or Bayerer's models [10]. The lifetime expectancy (in numbers of cycles) of an IGBT is experimentally obtained from the given environmental and load conditions [11]. The cumulative fatigue (or damage) of an IGBT is calculated based on actual operational conditions and its RUL which is then expressed as the number of cycles to failures. The third method is the failure precursor method. This is realized by observing changes in either fault-related parameters or by adding purpose-built embedded sensors. All these methods have advantages and drawbacks. The first method is technically effective but provides limited information of the device's life consumption. It is irreversible and also affects the system architecture of the chip [12]. The second can offer quantitative information of the RUL from exhaustive tests. However, these parameters are often associated with different stress conditions and are not fully considered. For instance, humidity and mechanical stress are key parameters but are not accounted for [6]. While the third method is indirect and easy to implement, the complex relationship between failures and precursors is oversimplified.

Overall, failure mechanisms have not been fully understood and these methods cannot be applied in service conditions [13].
TABLE II

IGBT DEGRADATION PHASE DURATION

\begin{tabular}{lcccc}
\hline \hline \multicolumn{5}{c}{ Duration of Each Degradation Phase } \\
\hline IGBT No & 1 & 2 & 3 & 4 \\
\hline 1st Phase & 1 & 2 & 2 & 2 \\
2nd Phase & 109 & 132 & 61 & 117 \\
3rd Phase & 1245 & 955 & 1069 & 1561 \\
4th Phase & 1440 & 1429 & 1940 & 1296 \\
5th Phase & 656 & 866 & 317 & 341 \\
6th Phase & 88 & 164 & 645 & 480 \\
7th Phase & 521 & 879 & 266 & 394 \\
8th Phase & 282 & 94 & 145 & 331 \\
9th Phase & 45 & 78 & 209 & 85 \\
10th Phase & 111 & 6 & 17 & 91 \\
IGBT Life & 4498 & 4605 & 4671 & 4698 \\
\hline \hline
\end{tabular}

This paper proposes a new data-driven approach for power electronic components (e.g., IGBT), which addresses issues in previous IGBT prognostics. In [14], Celaya et al. proposed an empirical degradation model for the $\Delta \mathrm{R}_{-}[\mathrm{DS}(\mathrm{ON})]$ as the degradation process for six aged devices. The exponential model is formed as a first-order linear discrete model for using Bayesian tracking algorithms like the extended Kalman filter. In [15], Patil et al. introduced a prognostics framework for nonpunch through and field stop IGBTs (NPT IGBTs), which includes the Mahalob distance anomaly parameter detection as the diagnostic parameter. The particle-filter is performed for future health prediction of the component using the $\mathrm{V}_{-}[\mathrm{CE}(\mathrm{ON})]$ as a precursor parameter. The first step in this approach is to propagate a number of particles through the empirical model to estimate the initial state. The second step is to estimate the next state based on the first updated particle weights.

The defect is dependent on the propagation by time steps (in all the above approaches). It is computationally unfeasible to perform several Monte-Carlo Simulations (MCS) at each time step. In the proposed novel prognostic algorithm, the dependency of the RUL efficiency on the time step is eliminated and MCS are carried out for the various degradation phase durations (see Table II). As a result, the RUL time consumption is significantly reduced and a considerably improved prognostic feasibility model is embeddable. The aim of this paper is to develop a computationally efficient and an embedded real-time prognostic approach. In this regard, we propose a novel probabilistic prognostic model for each degradation phase of the system for estimation of the health state of the IGBT component. RUL simulation is performed using the Monte-Carlo technique (multiple runs) of the degradation model up to the predefined end-of-life threshold. In this approach, the RUL calculation is carried out using the probabilistic model as a function of the component's life duration. This modeling technique uses the historical degradation data to construct the probability of failure during operating conditions. The dataset is discretized in various states which have different durations. Therefore, the probabilistic model is formed utilizing the duration of the failure phase in 
order to aid the model propagation, which effectively reduces the total computing processing.

This paper is organized as follows. In Section II, the phenomena of intrinsic and extrinsic failure mechanisms are described in brief and some of the failure modes applicable to power electronic components are discussed. The power cycling test rig and effects of power cycling are described in Section III. The data collection, data preprocessing, and classifications are then presented. Section IV discusses degradation models, problem formulation, and the model's degradation optimization. Section $\mathrm{V}$ discusses the prognostics approach using a statistical model. It also discusses the MCS method and the IGBT degradation model for RUL estimation. Section VI discusses the prognostics results using root mean square error (RMSE). Concluding remarks and future work are discussed in Section VII.

\section{FAILURE Mechanism OVERVIEW}

In order to model the reliability of power electronic components, it is necessary to understand the failure mechanisms and identify the precursors of failure, as well as of aging under various operating conditions and environmental stress to predict the malfunctioning of the component. The two major failure mechanisms of IGBTs are intrinsic and extrinsic faults that are related to IGBTs' physics and packaging, respectively. The phenomenon of intrinsic failure mechanism in power electronics includes hot carrier injection (HCI), latch up mechanism (i.e., sudden collapse of the collector to emitter voltage), dielectric breakdown, and electromigration. Extrinsic faults consist of wire lift off, die solder delamination, and substrate solder degradation [16], [17]. On the other hand, the aging of power electronic systems resulting from harsh operating conditions and environmental stress are two potential failure modes of stress and cause of catastrophic failure in IGBTs. As this paper intends to develop a prognostic model based on the precursor's parameters such as $v_{c e}$, $v_{g e}$, and $i_{c e}$ [18]-[22], it would be useful to understand the physics behind the failure mechanisms that can be monitored by such parameters (mainly $v_{c e}$ in this paper).

One of the most important defects relevant to electric stress is related to dielectric breakdown. This defect mechanism occurs in between the channels of the gate oxide, the emitter, and collector. It is noted that the gate oxide degrades when the dielectric layer wears out due to a strong electric field of $10 \mathrm{MV} / \mathrm{CM}$ or above and electrostatic discharge [23]. The gate structure includes a thin oxide layer, which is used to isolate the gate from the MOS transistor, which due to a large voltage spike, emits an avalanche of electrons which causes an immediate breakdown [24].

$\mathrm{HCI}$, an intrinsic failure mechanism, is considered as either being the primary cause of time-dependent dielectric breakdown or wearing out of chips under harsh operating conditions [25]. In a high electric field, the carriers (electron/hols) have sufficient energy to break through the $\mathrm{Si} / \mathrm{SiO}_{2}$ barrier, which causes leakage current through the oxide, whereas high temperature causes gate oxide breakdown. As a result of hot electron injection which leads to excessive leakage current, the IGBT's turning off time is effectively increased, which leads to a loss of both gate voltage and control of the collector current $\left(i_{c}\right)$. Hence, HCI has a major effect on the long-term reliability of power electronic components [18]. Another intrinsic failure phenomenon is electromigration failure, which might occur when the current is unevenly distributed among wire bonds. The reason is that the current density being much higher in the vicinity around some bonding wires together with an increase in temperature will cause an adjustment in the interconnection of the wire bonding eventually resulting in a break in the cross section of the wires. As a result, the conductor resistance and overall temperature of the devices are increased [26], [27].

\section{AGING EXPERIMENT}

In order to create a prognostic model, it is important to collect reliability information in the form of a degradation profile that covers failure data under various types of operating conditions. It takes a long time for failures to progress in actual operating conditions. It is thus necessary to employ a number of different techniques to accelerate failure mechanisms. These techniques, known as accelerated aging tests, are divided into three main categories: thermal cycling [11], [28]-[30], power cycling [31], [32], and electrical overstress [33]. It is noteworthy that due to the nature of failures, different failures are induced under different aging experiments. Hence, it is necessary to collect degradation information, which is accelerated by different types of accelerated aging tests. For example, wire bond failure is the dominant failure for IGBTs in relatively highly accelerated thermal and power cycling aging tests [34]. However, other mechanical failures, such as solder joints and metallization, can dominate the failure mode in lower temperature condition stress testing. The accelerated aging test is useful in identifying these dominant failures and concentrating our attention on these failures in power cycle testing. In this paper, the power cycling accelerated aging test rig was run by the Power Electronic Group at Nottingham University. As the test rig was run with a highly accelerated degradation process, the failure mode corresponds to the wire bond failure and eventually turns to latch up failure condition. Hence, the proposed prognostic model can predict these sorts of failures. The rest of this paper describes the test bench used by the Power Electronics Group and the data-mining steps, which are needed to prepare reliability data for the creation of the prognostic model.

\section{A. Power Cycling Aging Bench}

The Power Electronics Group at Nottingham University has carried out a power cycling aging test for IGBT power electronic devices under high thermal stress values up to $60^{\circ} \mathrm{C}$ for the baseplate temperature and $120{ }^{\circ} \mathrm{C}$ for the die. The failure mode involves wire bond lifting off and progressively ending before reaching the open circuit. The device is assembled using Dynex technology of the alumina-copper using direct bonded copper for heat conduction and relatively low conductivity of alumina, and aluminium wire bonds (see Fig. 2).

The test bench is equipped with a switched current supply and a constant current power supply to heat and cool a die sample. Each IGBT die is soldered and wire bonded onto a substrate tile. 


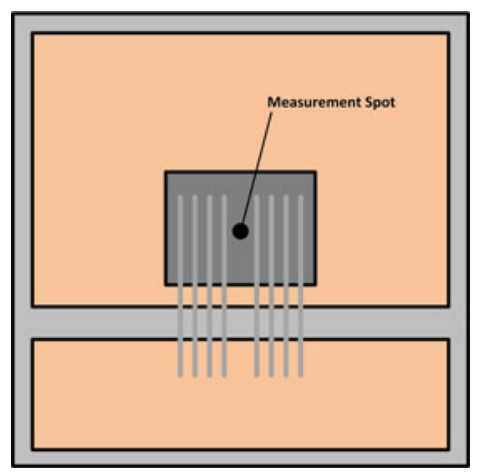

Fig. 2. Proposed Sample.

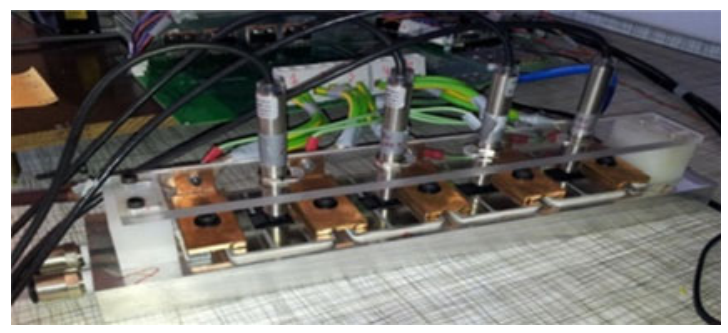

Fig. 3. Actual Heat Sink Assembly, IR Sensor Support, and IR Sensors.

A coupon is used to hold the die and to constantly dissipate heat through the heat sink and water coolant loop. This also allows the temperature to be measured using an infrared (IR) sensor.

The custom test bed provides a coolant at a constant $20^{\circ} \mathrm{C}$ using a chiller unit (Thermo FisherScientific A200-A25) and the coolant flows in a U-shape circuit through the heat sink. The four coupons sit in recessed areas on the upper surface of the heat sink. The supported IR sensors used above the coupons are Micro-Epsilon CS-SF15-C1 miniature pyrometers. These can be configured to output an analogue voltage, proportional to temperature, in the $0-5 \mathrm{~V}$ range using a USB programming kit (TM-USBK-CS USB Kit) (see Fig. 3).

The voltage signal from the IR sensors can also be used to visualize the temperature cycling in real time using Labview or similar software. Each coupon is allowed to heat up within the range of operating junction temperatures $\left(60^{\circ} \mathrm{C}\right.$ to $120^{\circ} \mathrm{C}$. Once the reading from the IR sensors indicates the temperature has reached the upper bound, then the bypass switch (designed to keep the temperature within a hysteresis bound) diverts heat away until the temperature drops to the lower limit. On the other hand, if the IR sensor indicates the temperature has fallen to the lower temperature bound, the bypass switch will be disabled and the cycle repeats until the safety temperature bound which is set to prevent the device from open-circuit failure. Such a test bench is needed to age the component under various operating conditions subjected to various mission profiles, which characterize the operational environment. It is expected to work under ambient conditions, and high-temperature cyclic, and intermittent loading conditions (i.e., power cycling), which ultimately induces more stress on the weak parts of the device [20].

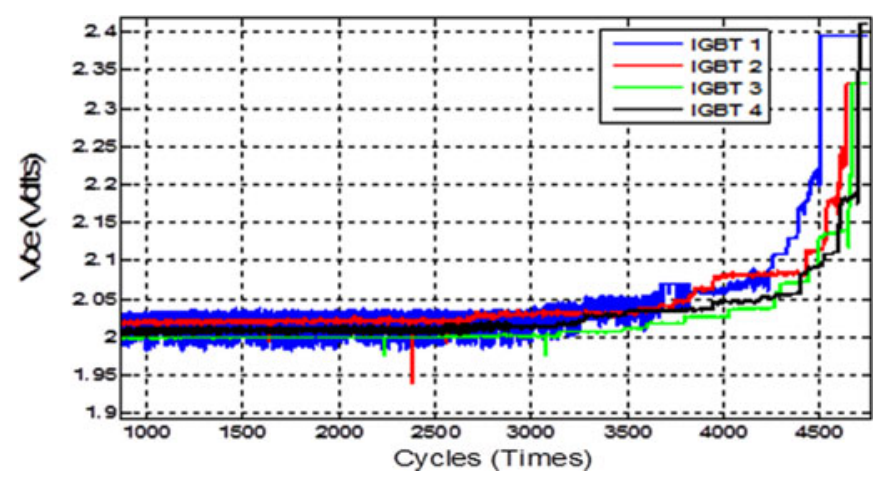

Fig. 4. Four IGBT run-to-failure dataset samples.

Power cycling aging tests enable monitoring and measurement of temperature and electrical parameters. The junction temperature and the collector-emitter are measured and recorded constantly until the IGBT fails in accelerated aging experiments. The dataset is formatted in a data array fashion, which can be read by MATLAB to employ reliability analysis on the raw data for conducting data preprocessing, as described in the following section.

\section{B. Data Preprocessing and Discretization}

Following the transfer of data to MATLAB, it is necessary to conduct preprocessing which makes the reliability information suitable for developing a prognostic model (i.e., data mining). Data mining comprises preprocessing (filtering), classification (discretizing), formulating a degradation model, and propagation (RUL simulation) [35], [36]. The $v_{c e}$ (on-state) parameter indicates increases in nonmonotone discrete steps including noise until the IGBT fails and before an open circuit occurs for all four samples (see Fig. 4). The $v_{c e}$ measurement enables the detection of wire bond lift off or emitter metallization damage [6], [9], [15]. The $v_{c e}$ variation due to the degradation process is very small and requires a very high degree of accuracy for this measurement. As shown in Fig. 4, the $v_{c e}$ (on-state) voltage precursor indicates a sudden fall at the end of the aging process when the IGBT fails after more than 4500 time units.

IGBT raw datasets have inherent noises which obscure the trend underlying the samples. Initially, the raw dataset is processed using a low-pass filter, which attempts to reduce internal noise as a dispersion sensitivity to reveal the true value of the aging data of raw $v_{c e}$. The drawback of this filter is that it imposes delays on the dataset. In Fig. 5, the green plot represents a low-pass filter with RC delay of 1000 time units; the magenta plot depicts a symmetrical low-pass filter; and the blue plot represents the raw data.

The symmetrical low-pass filter (i.e., moving averages filter) is proposed to overcome the lagging. The advantages of the moving average filter, as in (1), is that it results in more weighting of the population of data and less delay in filtering, and while minimizing noise, it effectively smooth's the dataset and can be a better use for data pattern recognition or classification as an 


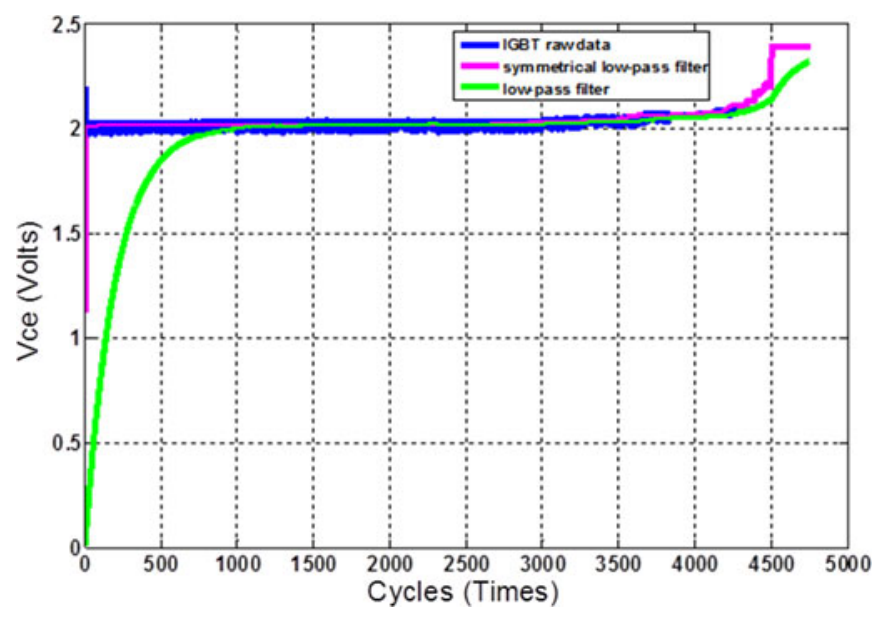

Fig. 5. First $v_{c e}$ sample after filtering.

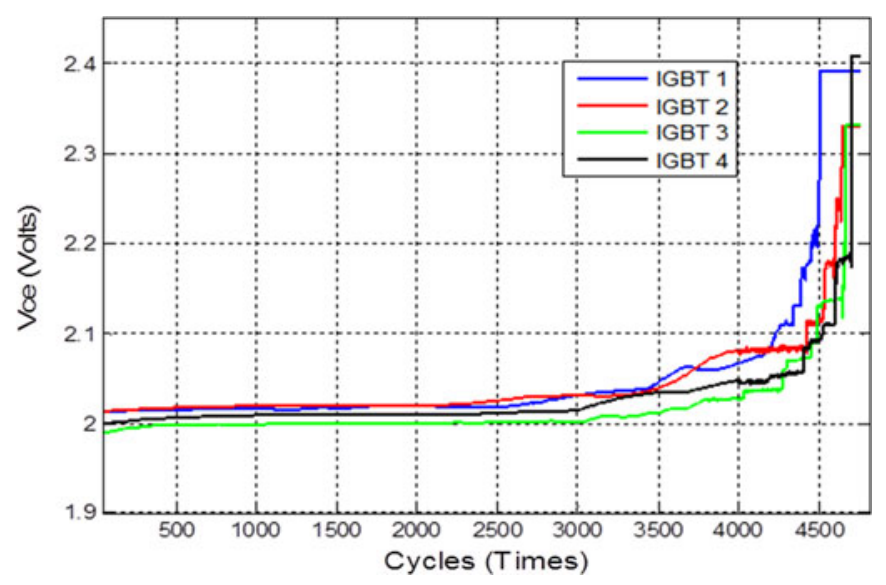

Fig. 6. Four IGBT run-to-failure datasets after filtering.

essential step for developing stringent prognostic modeling [37]

$$
\begin{aligned}
M A_{j} & =\sum_{i=j}^{n} \frac{x_{i}}{n-j} \\
n & =1000 \\
j & =1,2,2, \ldots, n-1 .
\end{aligned}
$$

The parameter $j$ denotes the reading points of both filtered and nonfiltered test data. $X_{i}$ is nonfiltered test data at reading point $i$. $n$ shows the length of the first relaxing point, which is varied from test data to test data. $n$ is set to 1000 for the data test shown in Fig. 5.

The rate of filtering windows increases when it reaches close to the relaxing point, whereas the length of the defined window becomes increasingly smaller. After noise elimination (see Fig. 6), the dataset is clean enough and suitable to be separated into different health states. Therefore, classification, an important part of the data-mining approach, is performed for accurate and efficient RUL computation.

It is noted in Fig. 6 that the aging process started at almost $2 \mathrm{~V}$ and degradation is progressed for more than 4500 cycles, whereas the failure has occurred at about $2.4 \mathrm{~V}$. The degradation

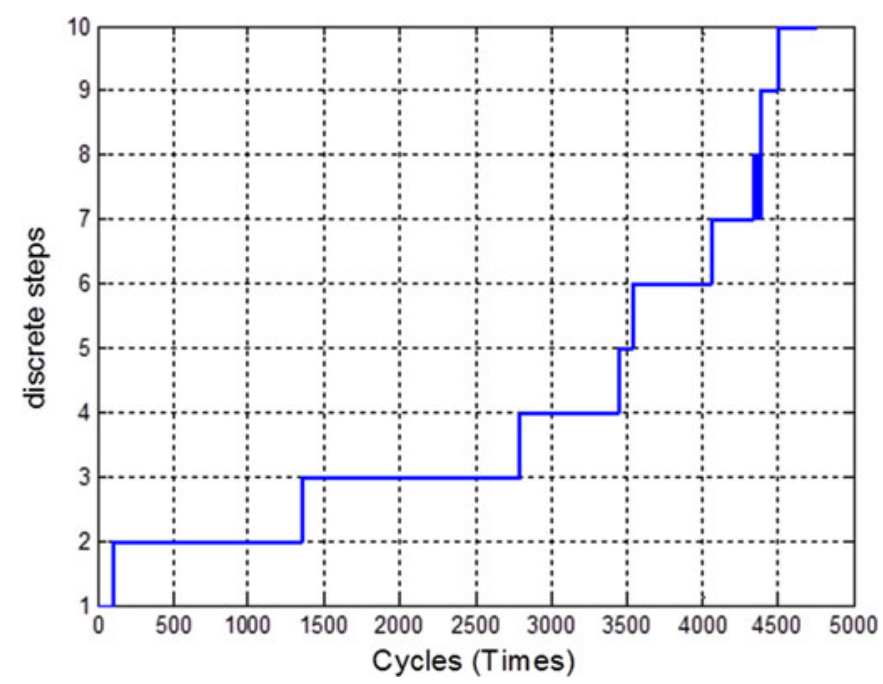

Fig. 7. Discretization of aging data.

process is discretized using a uniform quantization process [38] and each degradation state increases about $0.054 \mathrm{~V}$ in a discrete manner, which corresponds to one wire bond lift off. Fig. 7 depicts ten health states and each state lasts for a period of time before it reaches the subsequent state.

Fig. 8 depicts the overall process of the data-mining algorithm development for the IGBT $v_{c e}$ dataset collected from power cycling experiments. Initially, the aging data of raw $v_{c e}$ as a precursor parameter is processed by low-pass filtering. Then, the state of degradation is discretized in the form of the hidden health condition. The estimation model as a failure model (degradation model) is then structured from the training dataset using conventional statistical models (Gamma, Poisson, etc.). Subsequently, the trained model is used with the test data to estimate the current health of the component up until a predefined threshold state, where it fails gradually in a discrete manner. This information is then used with a MCS to predict the RUL of the IGBT. The outline of the algorithm is described in Fig. 8.

\section{Developing the Degradation Model}

\section{A. Problem Formulation}

As mentioned in the previous section, the noise-free aging data was discretized into different steps (phases). The phase durations of the run-to-failure of four IGBT samples are recorded after using a quantized cluster validity index and are displayed in Table II. The highest number of the failure progression occurs at the tenth health state [39].

A stochastic model is used in this paper because the occurrence of the IGBT degradation is assumed to have followed a nonhomogeneous probability distribution process, which renders the occurrence of a random event. As a result, the failure pattern follows the statistical approach in which the mean and standard deviation are the main parameters for tuning the probability functions (see Table III). Hence, the prognostics model can be formulated with statistical distribution models. 


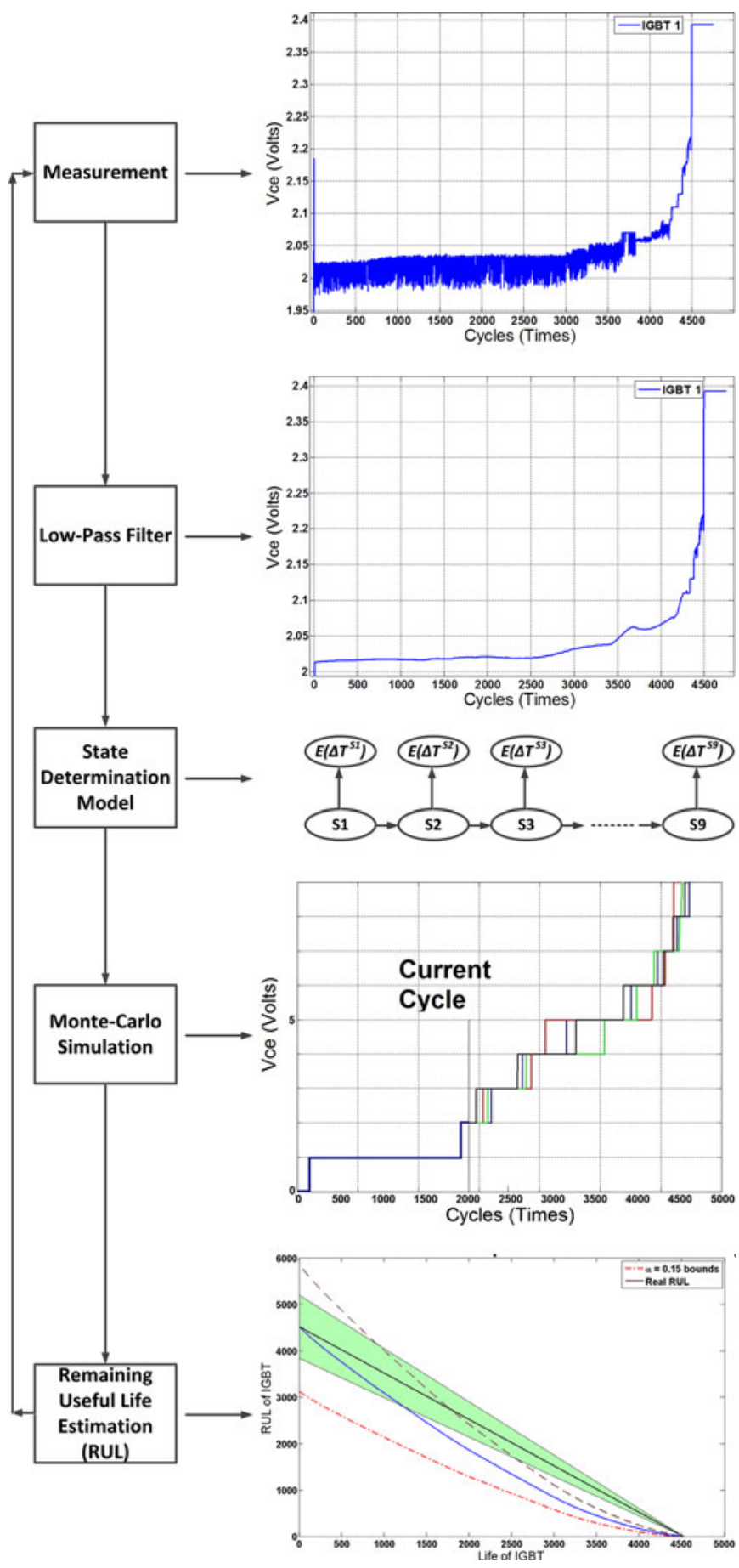

Fig. 8. Algorithm process.

In this paper, the histogram of the limited (four) run-to-failure samples will not provide the precise distribution. As such, two distributions have been selected: the Gamma and Poisson distributions. Two major types of distributions have been identified: 1) distributions which have mean $(\lambda)$ and standard deviation $(\delta)$, i.e., Gaussian, exponential, Weibull distributions, etc.; 2) distributions with solely $\delta=l$, i.e., Poisson, binomial distributions, etc. All the listed distributions in Type 1 will work in a similar manner and the same is true of Type 2 distributions. Therefore,
TABLE III

PROBABILITY DISTRIBUTION FUNCTION

\begin{tabular}{lcc}
\hline \hline Models & Density Functions & Parameters \\
\hline Gamma & $f\left(T_{i}=x\right)=x^{k-1}\left(e \frac{-x}{\theta} / \Gamma(k) \theta^{k}\right)$ & $\kappa, \theta$ \\
Poisson & $f\left(T_{i}=x\right)-\frac{e^{-\lambda_{\lambda}}}{k !}$ & $\lambda$ \\
\hline \hline
\end{tabular}

each distribution has been peaked up from both types randomly. The Gamma distribution has been chosen as it is associated with mean and standard deviation parameters, whereas the Poisson distribution involves only a mean parameter. Hence, this paper aims to present the feasibility of Gamma and Poisson models in problem formulation of prognostics. Their validity is evaluated in Section VI.

Both can be modeled into each individual degradation phase. The duration time $(\Delta T)$ of the degradation is considered to be a random variable, which can be a random parameter for the probability distribution function. The duration of each degradation process is used as a parameter $\lambda$ for the Poisson probability distribution function and $\kappa, \theta$ for the Gamma probability distribution function [39].

\section{B. Degradation Model Optimization}

Maximum likelihood estimation (MLE) is used as an objective function to maximize the density probability function (i.e., the Poisson distribution function). The Poisson distribution $\left(P\left(x_{i} \mid \lambda\right)=\left[\frac{\lambda^{x_{i}} \cdot e^{-\lambda}}{x_{i} !}\right], \quad x_{i} \geq 0\right)$ is the probability distribution of IGBT data $\left(x_{i}\right)$ given Poisson parameter $\lambda$. The MLE method is used to estimate the underlying rate parameter $(\lambda)$ for the Poisson process in order to generate the probability of occurrence for each $\lambda$. The first step is to write the joint probability mass function in this case because $x_{i}$ is a discrete random variable of positive integers. As all discrete random variables are independent, the product of individual density functions can be obtained

$$
P\left[x_{1}, x_{2}, \ldots, x_{n} \mid \lambda\right]=\prod_{n=1}^{N} \frac{\lambda^{x_{n}} e^{-e \lambda}}{x_{n} !}=c \cdot e^{-\lambda_{N}} \cdot \lambda^{\sum_{n=1}^{N} x_{n}} .
$$

The next step would be to calculate the maximum of this probability mass function with respect to $\lambda$. This can be done by taking a logarithm, which simplifies the product of exponents. This is equivalent to finding $\operatorname{Max} \lambda$ [40]

$$
\log \left(c \cdot e^{-\lambda_{N}} \cdot \lambda^{\sum_{n=1}^{N} x_{n}}\right)=-N \lambda+\left(\sum_{n=1}^{N} x_{n}\right) \cdot \ln \lambda .
$$

Then, taking the derivative from (4) and setting it to zero $\left(\frac{\partial}{\partial \lambda}=0\right)$ produces $\hat{\lambda}$ MLE [24]

$$
\hat{\lambda} \mathrm{MLE}=\frac{1}{n} \sum_{n=1}^{N} x_{n} .
$$


TABLE IV

MLE FOR THE POISSON PROBABILITY DISTRIBUTION

\begin{tabular}{lc}
\hline \hline MLE Model & Parameters \\
\hline & $\lambda$ \\
Model 1 & 2 \\
Model 2 & 98 \\
Model 3 & 1210 \\
Model 4 & 1531 \\
Model 5 & 520 \\
Model 6 & 330 \\
Model 7 & 520 \\
Model 8 & 211 \\
Model 9 & 126 \\
Model 10 & 70 \\
\hline \hline
\end{tabular}

TABLE V

MLE FOR GaMma PROBABILITy DistribUTION

\begin{tabular}{lcc}
\hline \hline & \multicolumn{2}{c}{ Parameters } \\
\cline { 2 - 3 } MLE Model & $\kappa$ & $\theta$ \\
\hline Model 1 & 0.6646 & 694.9 \\
Model 2 & 0.6020 & 766.9 \\
Model 3 & 0.4284 & 1053.3 \\
Model 4 & 0.3378 & 978.3 \\
Model 5 & 0.3086 & 576.5 \\
Model 6 & 0.2692 & 458.4 \\
Model 7 & 0.2389 & 372.9 \\
Model 8 & 0.2456 & 153.0 \\
Model 9 & 0.2578 & 63.3 \\
Model 10 & 0.2964 & 20.0 \\
\hline \hline
\end{tabular}

For the Gamma distribution, there are two modeling parameters, $\kappa$ and $\theta$, to be estimated. MLE generically is formulated as

$$
\hat{\theta}=\frac{1}{\kappa N} \sum_{i=1}^{N} x_{i} .
$$

The maximum likelihood criterion is a fairly general one, and also, fairly powerful to show the true value (the maxima) in close form algebraically. The true maximum likelihood estimator $\hat{\theta}$ and $\hat{\lambda}$ MLE parameters converge to the true parameter value $\lambda$. Using the analyticalMLE method, estimation of the best fit of the modeling parameter $\lambda$ for tuning the Poisson distribution is performed [41] (see the estimated values in Table III). Tables IV and $\mathrm{V}$ summarize the parameters for ten uncorrelated degradation phases obtained from MLE of the Poisson and Gamma distributions, respectively.

In Fig. 9, the black discrete plot is the optimized parameters of all four IGBT samples, using the MLE function, which has shown the tracking of the trend of the first IGBT sample as an example.

\section{PROGNOSTIC APPROACH}

In this section, an overview of the prognostic model learning is provided. This is followed by details of RUL estimation in the following sections.

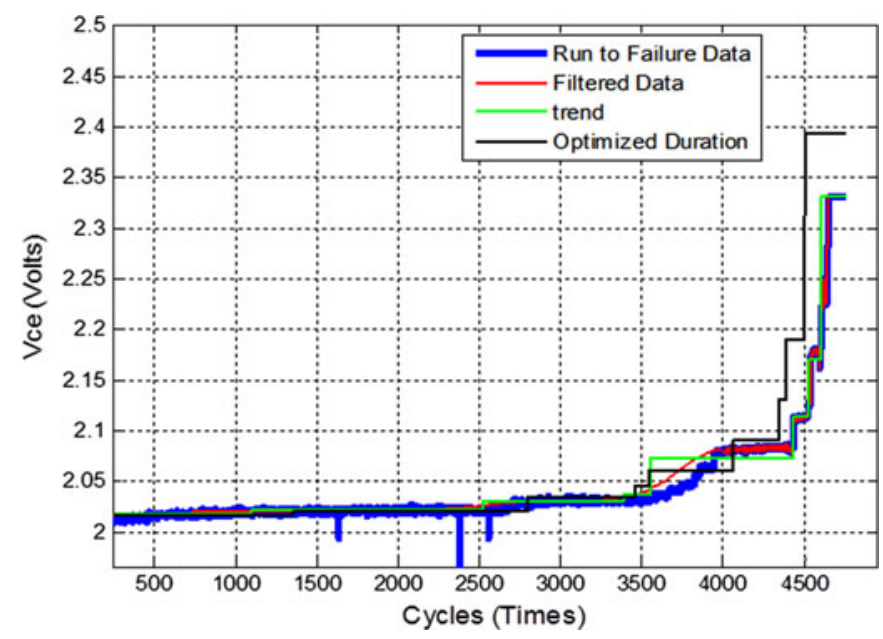

Fig. 9. Estimated parameter tracks the trend of the IGBT dataset.

\section{A. Model Learning}

In this section, the prognostics algorithm is described in detail and is shown in Fig. 10. Feature extraction (i.e., duration), clustering, and duration optimization using MLE functions are obtained before model estimation. The threshold value must therefore be defined for the propagation stage. In this regard, the ninth state which almost indicates the end of the component's life is defined as a threshold state. This is an essential step for the data-mining process, which the degradation model propagates up until the predefined threshold state.

A cross validation technique is used to assess the accuracy of the predictive degradation model (Poisson and Gamma models). Therefore, in this paper, the first three IGBT samples are chosen as the training dataset and the fourth as the test data. In the next iteration, the first training dataset is shifted to the second training dataset and the second training dataset is shifted to the third and so on, until the first one reaches the last dataset. The test data will then be rotated one by one for all four IGBT datasets. The probability distribution function (e.g., Poisson function) is used to estimate the expected value using mean parameter $\lambda$ value. Nine stochastic models are built based on nine degradation phases, which look similar to a Markov model structure as shown in the following equation:

$$
\frac{1}{N} \sum_{i=1}^{4} \sum_{j=1}^{9} \Delta T_{\left(S_{j}\right)}^{(j)}=E\left(\Delta T^{S_{j}}\right) .
$$

After the states are parameterized by mean duration $E\left(\Delta T^{S_{j}}\right)$ for all degradation phases (see Fig. 11), the degradation model based on stochastic probability density functions (Poisson probability distribution) are constructed in the form of a Markov model structure. Subsequently, the next step is to calculate the end of life of the component [42].

\section{B. RUL Estimation}

In this paper, the feasibility of RUL estimation presents as the useful life left on an IGBT component at a particular time of measurement. The approach depends on past observed IGBT 


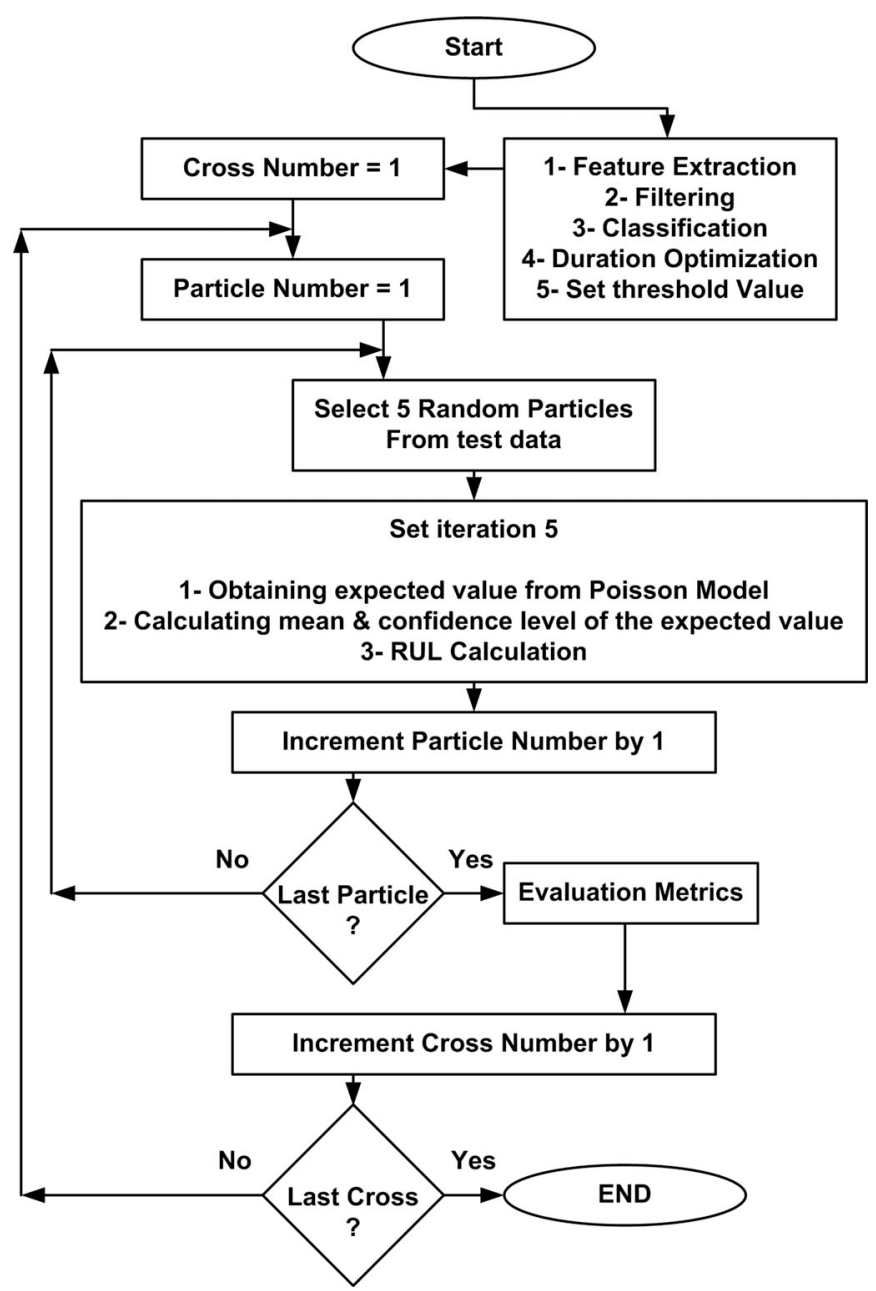

Fig. 10. Prognostic approach algorithm.

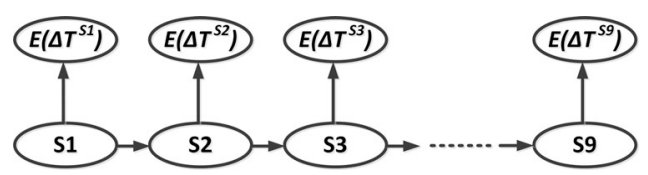

Fig. 11. Degradation model's structure for prognostics.

degradation data, which is processed to construct an estimated model based on the duration parameter of the failure data using the Poisson and Gamma distribution functions. Both Poisson and Gamma models are conducted with the same RUL estimation format. Due to this similarity, only the RUL estimation for the Poisson process is described. However, while both are conducted with the same process, the results for both emphasized differences in RUL calculations which will be explained in the next section.

The degradation model is constructed using the Markov approach and MCS are used to estimate the value up to a predefined threshold state [43]. Then, the RUL (i.e., mean, median, and confidence bounds) is calculated using the distribution of simulated RULs based on the duration of the degradation phase. First, the particle is peaked up from the selected dataset and propagated

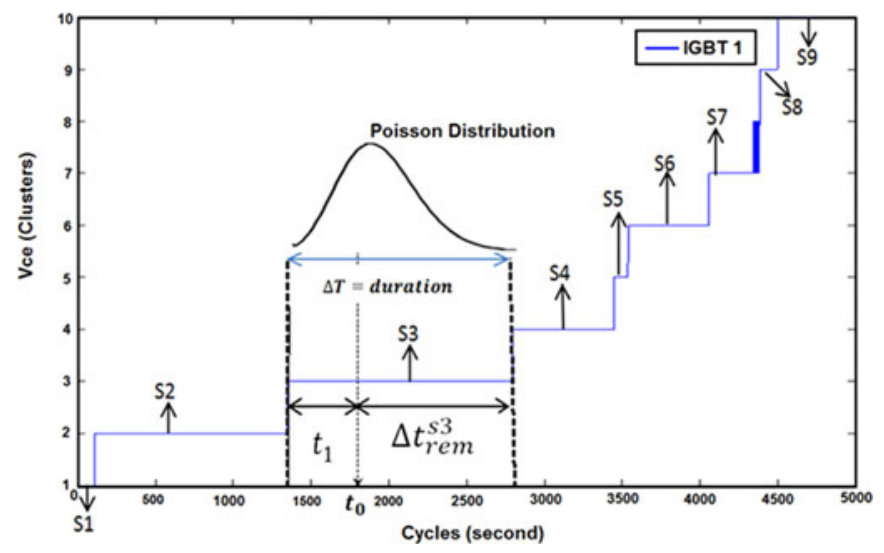

Fig. 12. Constructed Markov model and MCS for RUL calculation.

through the degradation model up until the predefined threshold state 9 . The propagation is iterated five times according to the algorithm (see Fig. 10).

Before incrementing at a particle number, the mean of the expected value is calculated using MATLAB command Poisstat, and subsequently, using Poissfit to return a 90 percentile confidence level of the optimized parameter. The ten percentile bounds of the expected value are also calculated using Poissinv. To successfully estimate the RUL of the IGBT component, this process will continue up until the last particle of the selected test dataset is greater than the length of the test data and if not, the particle is incremented by one, the same process is repeated for all data points. Subsequently, the process moves to calculate the prognostic evaluation metric using the RMSE method. Cross validation is performed to evaluate the prognostic results by changing the test sample one by one after the RMSE value is calculated for each individual test sample. The RUL of the component life is calculated using the operation time (opt) measured at $t_{0}$, using (6), e.g., $\mathrm{IGBT}_{1}$ shown in Fig. 12 and [44]

$\operatorname{RUL}\left(\mathrm{IGBT}_{1}\right)=$ component life $\left(\mathrm{igbt}_{1}\right)$-operation time $\left(t_{0}\right)$

$$
\begin{aligned}
\text { Component life }\left(\text { igbt }_{1}\right)= & t_{0}+\Delta t_{r e m}^{s 3}+\Delta t_{4}^{s 4}+\Delta t_{5}^{s 5} \\
& +\Delta t_{6}^{s 6}+\Delta t_{7}^{s 7}+\Delta t_{8}^{s 8}+\Delta t_{9}^{s 9}
\end{aligned}
$$

where $\Delta T$ is generated by MCS, which presents the duration of the relevant degradation phase. It follows the model distribution based on the duration of the degradation phase, and it is optimized using the MLE function. As noted from Fig. 12, $t_{1}$ is the time of the last degeneration phase and it will move to the next degeneration phase. If the random value $\Delta t_{r e m}^{s 3}$ is equal to $\left(\mathrm{E}\left[\Delta t^{s 3}\right]=\Delta T\right)$ for duration $\Delta T \leq t_{1}$, then the random value $\left(\Delta t_{\text {rem }}^{s 3}\right)$ would be equal to 0 . In case of $\Delta T \geq t_{1}$, $\Delta t_{r e m}^{s 3}$ is equal to $\Delta T-$. This means the component's health state is still in this degradation phase and it also continues in the same degradation phase. 

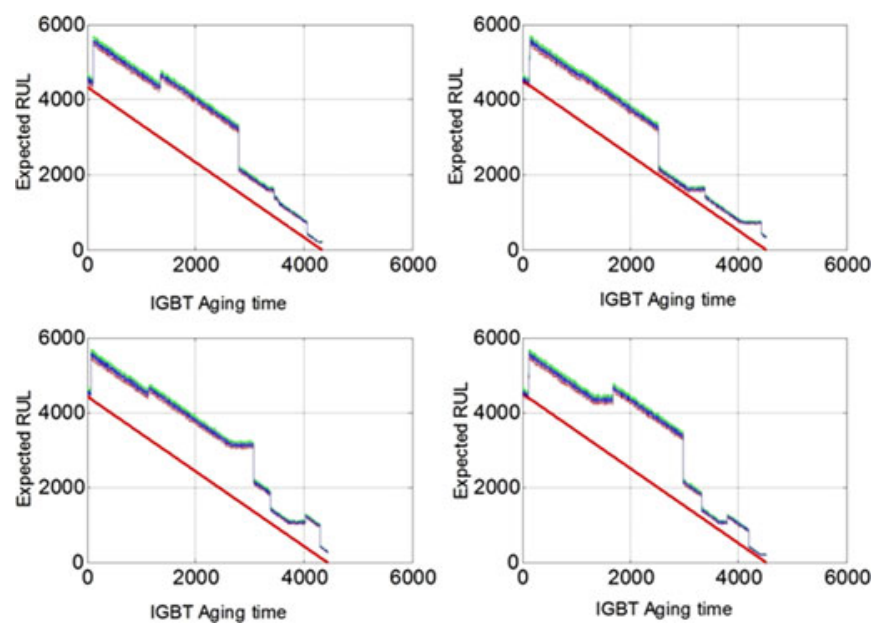

Fig. 13. RUL prediction results using the Poisson distribution.

\section{RESULTS}

For both estimation models, the prognostic methods have tested all four IGBT datasets and the results are depicted by a series of polylines. In this process of calculation of the real RUL of the IGBT, at the beginning, the convergence of the RUL estimation is high and it will decrease toward the end of the real RUL. At each measurement point, the degradation phase of the IGBT is predicted and the end of the degradation process is forecasted.

\section{A. Results From RUL Estimation Using Poisson Process}

Fig. 13 shows the IGBT life prediction results, which are validated with test samples using the cross validation technique. For the prognostic calculation using a Poisson distribution model, the straight red line represents the real RUL. The scattered red and green plots are used as $\pm 10 \%$ confidences levels, respectively. The scattered blue line is the mean value of the RUL prediction. They are used as baselines to show the MCS results during the degradation process.

It can be noted that the 90 and 10 percentiles confidence width bonds are very narrow and unrealistically close to the mean during the whole prediction and do not provide meaningful information for decision makers due the fact that the Poisson distribution does not have a standard deviation parameter. At the beginning of the execution, the mean is quite divergent and linear with sudden changes reflecting the number of discrete changes in the degradation phase, but slowly becomes convergent with the real value once the $v_{\text {ce }}$ measurement is updated and reaches the end of the component's life.

\section{B. Results From RUL Estimation Using Gamma Process}

In contrast to the Poisson process, initial results for the Gamma process were populated with uncertainity which provides meaningless information for the decision maker. The reason for this controversy is the Poisson distribution's mean value $(\lambda)$ is equal to the standard deviation $(\delta)$, which causes confidence intervals to overlap with the mean value. As a result,

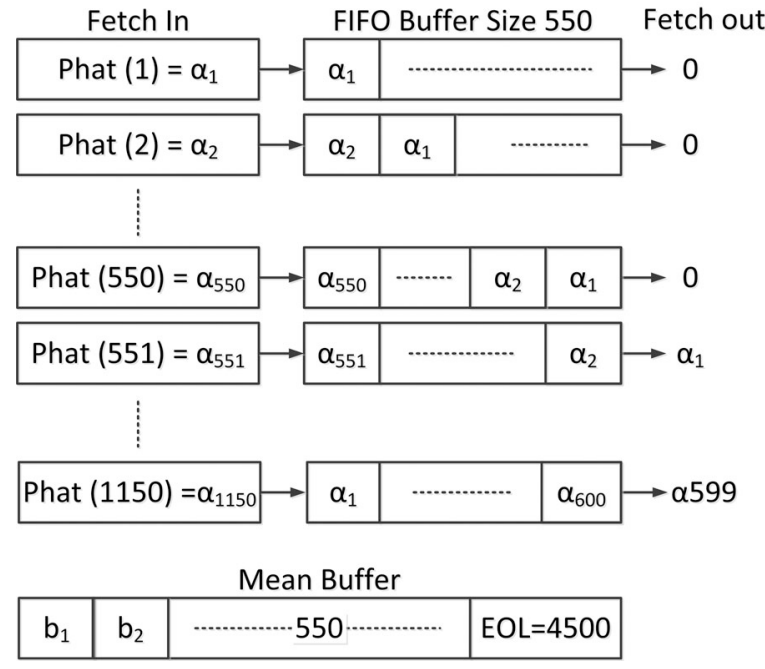

Fig. 14. Implementing FIFO buffer for prognostics approach.

uncertainty is trapped in between the narrow confidence bonds and does not appear in simulation results. The advantage of the Gamma-based estimation model is to have such a large confidence bound, which provides meaningful information for decisionmakers in comparison with the Poisson process. To resolve the problem of uncertainty, and to make the Gamma-based RUL estimation more appropriate for the decision maker, we propose FIFO (i.e., first-in first-out) buffering.

\section{FIFO Buffering for Gamma Approach}

A FIFO buffer (i.e., fist-in first out basis) is a useful way to store and smooth the estimated values using averaging FIFO buffer sizes. This will reduce uncertainty for accurate RUL estimation and improved confidence bounds. Conventional estimation models are relatively inefficient in that they reserve a large amount of uncertain data and unwanted information and this will propagate for the whole RUL calculation and become stale. This leads to ineffective prefetching when the Monte-Carlo method simulates the RUL estimation. We propose an alternative structure to store an estimation value in a predefined FIFO buffer size (e.g., 550 buffer size). All estimated data are placed at the bottom of the buffer and removed from the top. The mean buffer is maintained in direct correlation with the FIFO buffer. Thus, this method reduces stale uncertainty data, and allows a more accurate reconstruction of Monte-Carlo RUL simulation.

The MLE estimation value of the probability distribution function (e.g., Gamma, Weibull distributions, etc.) is returned in a vector (i.e., phat), which contains elements $\left\{a_{1}, a_{2}, a_{3}, \ldots, a_{n}\right\}$ and fetches into a FIFO buffer where the size of the buffer is optional. However, if the buffer size extends to the length of the component life, then the excess buffering of estimated RUL causes high latency, where the RUL estimation simulation deviates from the real RUL at the beginning (see Fig. 15). It can be noted in Fig. 14 that the first element fetches into the FIFO buffer frame and has shifted the next part when the second element arrives, and will continue until all 

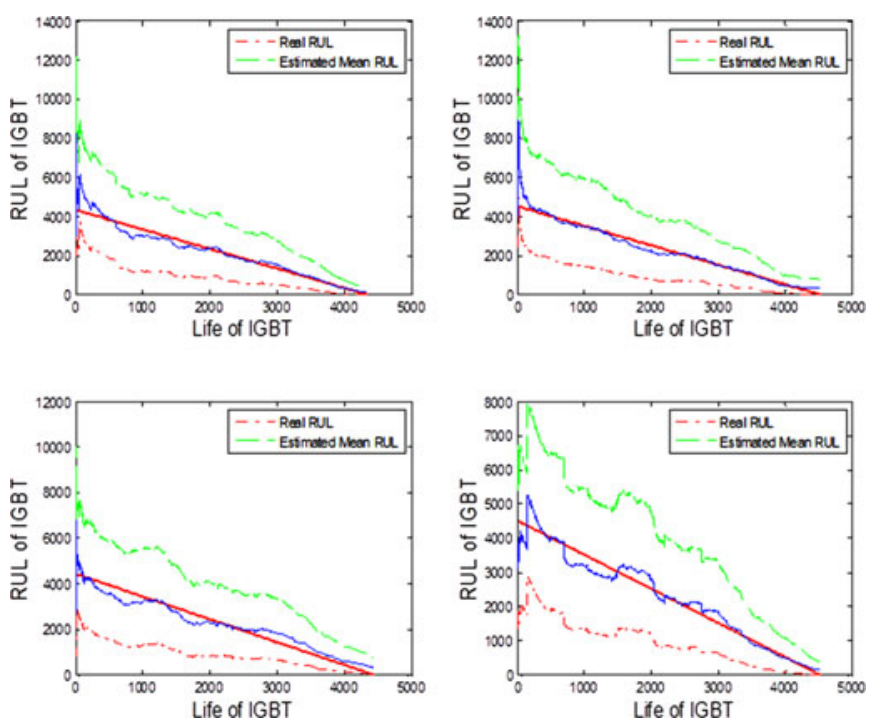

Fig. 15. RUL prediction results using Gamma distribution.

buffer sizes (550) are occupied with estimation elements. Simultaneously, the mean buffer keeps recording the average of the FIFO buffer elements as it increases (see (8) and (9)). Once it becomes bigger than the size of the buffer, the first element fetches out. Additionally, averaging of the FIFO buffer creates a single number at the end of the buffering that represents the typical distance, where the buffer sample is from the average. This phenomenon is essentially equivalent to the dc offset (i.e., mean value). This may affect the estimated RUL. Hence, the dc offset must be eliminated to reduce the convergence time. The elimination of the dc value is can be represented by $\mu$-min $(\mu)$, where $\mu$ is mean value

$$
\begin{aligned}
\text { If buffer size } & =S \leq 550 \Rightarrow\left\{b_{1}, b_{2}, b_{3}, \ldots, b_{s}\right\} \\
& =\frac{\sum_{n=1}^{n=s} a_{n}}{s} \\
\text { If buffer size } & =S>550 \Rightarrow\left\{b_{1}, b_{2}, b_{3}, \ldots, b_{s}\right\} \\
& =\frac{\sum_{n=(s-550)}^{n=s} a_{n}}{s} .
\end{aligned}
$$

Fig. 15 shows four IGBT samples of RUL prognostics results. The results are computed based on a Gamma distribution model with implementation of the FIFO buffer. As can be seen in Fig. 15, the results are promising for early failure findings and improve decision making based on $\pm 10 \%$ confidence levels. The straight red dash lines are used as the real RUL values. This is used as the baseline to indicate how well the prognostic algorithm performs during the test. In Fig. 15, the blue scatter plots are the mean value of the RUL prediction. The green and red plots are the 90 and 10 percentiles of the MCS degradation paths. At the beginning of the rendering test, the RUL prediction is slightly higher than the real RUL value. However, the predicted RUL slowly converges to the real RUL values as the operating time reaches the end of the component's life.
TABLE VI

Prognostic Performance Metric of Poisson Distribution

\begin{tabular}{lcccc}
\hline \hline $\begin{array}{l}\text { RUL estimation } \\
\text { metric }\end{array}$ & IGBT 1 & IGBT 2 & IGBT 3 & IGBT 4 \\
\hline RMSE\% & 0.286 & 0.200 & 0.258 & 0.272 \\
\hline \hline
\end{tabular}

TABLE VII

Prognostic Performance Metric of Gamma Distribution

\begin{tabular}{lcccc}
\hline \hline $\begin{array}{l}\text { RUL estimation } \\
\text { metric }\end{array}$ & IGBT 1 & IGBT 2 & IGBT 3 & IGBT 4 \\
\hline RMSE\% & 0.3317 & 0.3397 & 0.3485 & 0.3319 \\
\hline \hline
\end{tabular}

\section{Prognostic Evaluation Metric}

The difference between the estimated value and the real RUL is considered as an error and the standard deviation of the error is recognized as the RMSE value. This prediction metric renders the accuracy and precision for all prognostic methods. Therefore, in this paper, the RMSE is used to evaluate the prognostic results of the Poisson and Gamma distribution models [45]. A smaller value for RMSE means a more accurate result is produced by the prognostic model. If the $x_{\hat{i}}$ is the estimated and $x_{i}$ is the real value, then RMSE can be calculated using the following equation:

$$
\operatorname{RMSE}=\sqrt{\frac{1}{n} \sum_{i=1}^{n}\left(x_{\hat{i}}-x_{i}\right)^{2}} .
$$

Tables VI and VII summarize the mean-based RMSEs of the Poisson and Gamma distribution models of the four IGBT samples, respectively. The Poisson model has lower RMSE values in comparison to the Gamma model. Comparing the RMSE of the predicted RULs, the IGBT test sample number 2 has the smallest RMSE value for the Poisson model, whereas the IGBT test sample number 1 has the smallest RMSE value for the Gamma model. The rest of the other IGBT test samples have similar prognostics results.

\section{CONCLUSION}

In this paper, the prognostic algorithm represents a MonteCarlo RUL simulation and is based on a Markov stochastic duration model using the Poisson and Gamma distribution functions. The $v_{c e}($ on) is chosen as the best degradation indicator and precursor parameter. A degradation profile is obtained using the duration of the degradation process and the degradation phase is parameterized for the probability distribution function to be integrated in the prognostics algorithm for RUL estimation. It can be seen that the algorithm is capable of recalculating the RUL based on the measurement updates and merging it with the real RUL profile. The RMSE result indicates a good prognostic performance using the cross validation technique. In contrast to the Poisson process, the Gamma process needs FIFO buffering for the improvement of the prognostics results as uncertainty was an issue with the Gamma process. In contrast to current 
state-of-the-art prognostics, this paper represents a light-weight simulation-based prognostic approach because the RUL calculation takes less time, about $0.3 \mathrm{~ms}$ for each measurement point. Therefore, it could be implemented efficiently in a real-time prognostic calculation and is capable of providing an advanced failure warning and preventing costly power electronic system downtimes and failures.

\section{ACKNOWLEDGMENT}

The authors would like to thank Prof. C. M. Johnson and Dr. P. Evans from the Power Electronics, Machines, and Control Group, University of Nottingham, for the contribution of failure data of the IGBTs and the power cycling test rig configuration.

\section{REFERENCES}

[1] I. A. Khan, "Power electronics in automotive electrical systems," in Proc. IEEE Power Electron. Transp. Conf., pp. 29-38, 1996.

[2] N. Patil, S. Menon, D. Das, and M. Pecht, "Anomaly detection of non punch through insulated gate bipolar transistors (IGBT) by robust covariance estimation techniques," in Proc. 2nd Int. Rel., Safety Hazard Conf., 2010, pp. 68-72.

[3] R. W. Johnson, J. L. Evans, P. Jacobsen, J. R. Thompson, and M. Christopher, "The changing automotive environment: High-temperature electronics," IEEE Trans. Electron. Packag. Manuf., vol. 27, no. 3, pp. 164-176, Jul. 2004.

[4] R. Schmidt, F. Zeyss, and U. Scheuermann, "Impact of absolute junction temperature on power cycling lifetime," in Proc. 15th Int. Eur. Power Electron. Appl. (EPE), Conf., pp. 1-10, 2013.

[5] P. J. King and K. J. Burnham, "Use of confidence limits in the setting of on-board diagnostic thresholds," in Proc. UKACC Int. Conf. Control, 2012, pp. 708-712.

[6] B. Ji, V. Pickert, W. Cao, and B. Zahawi, "In situ diagnostics and prognostics of wire bonding faults in IGBT modules for electric vehicle drives," IEEE Trans. Power Electron., vol. 28, no. 12, pp. 5568-5577, Dec. 2013.

[7] J. M. Anderson, R. W. Cox, and P. O'Connor, "Online algorithm for early stage fault detection in IGBT switches," in Proc. 9th IEEE Int. Symp. Diagn. Electr. Mach., Power Electron. Drives, 2013, pp. 1-8.

[8] J. Gu and M. Pecht, "Predicting the reliability of electronic products," in Proc. 8th Int. Electron. Packag. Technol. Conf., 2007, pp. 1-8.

[9] B. Ji, V. Pickert, B. Zahawi, and M. Zhang, "In-situ bond wire health monitoring circuit for IGBT power modules," in Proc. 6th IET Int. Power Electron. Mach. Drives Conf., 2012, pp. 1-6.

[10] I. F. Kovacčević, U. Drofenik, and J. W. Kolar, "New physical model for lifetime estimation of power modules," in Proc. Int. Power Electron. Conf., 2010, pp. 2106-2114.

[11] P. James and A. Forsyth, "Accelerated testing of IGBT power modules to determine time to failure," in Proc. 5th IET Int. Power Electron., Mach. Drives Conf., 2010, pp. 1-4.

[12] E. E. Kostandyan and K. Ma, "Reliability estimation with uncertainties consideration for high power IGBTs in $2.3 \mathrm{MW}$ wind turbine converter system," Microelectron. Rel., vol. 52, no. 9/10, pp. 2403-2408, 2012.

[13] A. Kabir, C. Bailey, H. Lu, and S. Stoyanov, "A review of data-driven prognostics in power electronics," in Proc. 35th Int. Spring Semin. Electron. Technol., 2012, pp. 189-192.

[14] J. R. Celaya, A. Saxena, C. S. Kulkarni, S. Saha, and K. Goebel, "Prognostics approach for power MOSFET under thermal-stress aging," in Proc. Annu. Rel. Maintainability Symp., 2012, pp. 1-6.

[15] N. Patil, D. Das, and M. Pecht, "A prognostic approach for non-punch through and field stop IGBTs," Microelectron. Rel., vol. 52, no. 3, pp. 482-488, 2012.

[16] G. Sonnenfeld, K. Goebel, and J. R. Celaya, "An agile accelerated aging, characterization and scenario simulation system for gate controlled power transistors," IEEE AUTOTESTCON Conf., pp. 208-215, 2008.

[17] J. M. Anderson and R. W. Cox, "On-line condition monitoring for MOSFET and IGBT switches in digitally controlled drives," in Proc. IEEE Energy Convers. Congr. Expo., 2011, pp. 3920-3927.

[18] N. Patil, J. Celaya, D. Das, K. Goebel, and M. Pecht, "Precursor parameter identification for insulated gate bipolar transistor (IGBT) prognostics," IEEE Trans. Rel., vol. 58, no. 2, pp. 271-276, Jun. 2009.
[19] N. Patil, D. Das, K. Goebel, and M. Pecht, "Identification of failure precursor parameters for insulated gate bipolar transistors (IGBTs)," in Proc. Int. Conf. Prognostics Health Manag., Denver, CO, USA, 2008, pp. 1-5.

[20] M. A. Rodríguez-Blanco, A. Claudio-Sánchez, D. Theilliol, L. G. Vela-Valdés, P. Sibaja-Terán, L. Hernández-González, and J. Aguayo-Alquicira, "A failure-detection strategy for IGBT based on gate-voltage behavior applied to a motor drive system," IEEE Trans. Ind. Electron., vol. 58, no. 5, pp. 1625-1633, May 2011.

[21] V. Smet, F. Forest, J.-J. Huselstein, F. Richardeau, Z. Khatir, S. Lefebvre, and M. Berkani, "Ageing and failure modes of IGBT modules in hightemperature power cycling," IEEE Trans. Ind. Electron., vol. 58, no. 10, pp. 4931-4941, Oct. 2011.

[22] B. Saha, J. R. Celaya, P. F. Wysocki, and K. F. Goebel, "Towards prognostics for electronics components," in Proc. IEEE Aerosp. Conf., 2009, pp. 1-7.

[23] M.-D. Ker, "ESD (Electrostatic Discharge) protection design for nan electronics in CMOS technology," in Proc. Adv. Signal Process., Circuits, Syst. Des. Tech. Commun. Conf., 2006, pp. 217-279.

[24] M. Ciappa, "Selected failure mechanisms of modern power modules," Microelectron. Rel., vol. 42, no. 4/5, pp. 653-667, 2002.

[25] S. Yang, D Xiang, A. Bryant, P. Mawby, L. Ran, and P. Tavner, "Condition monitoring for device reliability in power electronic converters: A review," IEEE Trans. Power Electron., vol. 25, no. 11, pp. 2734-2752, Nov. 2010.

[26] H.-K. Tseng and M.-L. Wu, "Electro-thermal-mechanical modeling of wire bonding failures in IGBT," in Proc. 8th Int. Microsyst., Packag., Assem. Circuits Technol., Conf., 2013, pp. 152-157.

[27] R. Wu, F. Blaabjerg, H. Wang, M. Liserre, and F. Iannuzzo, "Catastrophic failure and fault-tolerant design of IGBT power electronic convertersAn overview," in Proc. IEEE 39th Annu. Ind. Electron. Soc., 2013, pp. 507-513.

[28] F. Wakeman, D. Hemmings, W. Findlay, and G. Lockwood, Pressure Contact IGBT, Testing for Reliability. Biel, Switzerland, IXYS Corp., 2002.

[29] A. Morozumi, Y. Nishimura, and S. Okita, "Semiconductor device and method of relaxing thermal stress," U.S. Patent 6844621 B2, Jan. 18, 2005.

[30] J. R. Celaya, P. Wysocki, V. Vashchenko, S. Saha, and K. Goebel, "Accelerated aging system for prognostics of power semiconductor devices," in Proc. IEEE AUTOTESTCON Conf., 2010, pp. 1-6.

[31] M. Mermet-Guyennet, X. Perpiñá, and M. Piton, "Revisiting power cycling test for better life-time prediction in traction," Microelectron. Rel., vol. 47, no. 9-11, pp. 1690-1695, 2007.

[32] A. Hensler, J. Lutz, M. Thoben, and J. Zachariae, "Power cycling tests at high temperatures with IGBT power modules for hybrid electrical vehicle applications," in Proc. 3rd Int. Electron. Syst.-Integr. Technol., Conf., 2010, pp. 1-6.

[33] S. Saha, J. R. Celaya, V. Vashchenko, S. Mahiuddin, and K. F. Goebel, "Accelerated aging with electrical overstress and prognostics for power MOSFETs," in Proc. IEEE Energytech Semin., 2011, pp. 1-6.

[34] M. Musallam, C. M. Johnson, C. Yin, H. Lu, and C. Bailey, "Real-time life expectancy estimation in power modules," in Proc. 2nd Int. Electron. Syst.-Integr. Technol., Conf., 2008, pp. 231-236.

[35] P. Baraldi, F. Cadini, F. Mangili, and E. Zio, "Model-based and data-driven prognostics under different available information," Prob. Eng. Mech., vol. 32, pp. 66-79, 2013.

[36] K. Goebel, B. Saha, A. Saxena, N. Mct, and N. Riacs, "A comparison of three data-driven techniques for prognostics," in Proc. 62nd Meet. Soc. Fail. Prev. Syst. Availability, 2008, pp. 119-131.

[37] P. Reyes, P. Reviriego, J. A. Maestro, and O. Ruano, "New protection techniques against SEUs for moving average filters in a radiation environment," IEEE Trans. Nucl. Sci., vol. 54, no. 4, pp. 957-964, Aug. 2007.

[38] S. Marano, V. Matta, and P. Willett, "Some approaches to quantization for distributed estimation with data association," IEEE Trans. Signal Process., vol. 53, no. 3, pp. 885-895, Mar. 2005.

[39] L. Ma, J.-S. Kang, C.-Y. Zhao, and S.-Y. Liu, "Modeling the impact of prognostic errors on CBM effectiveness using discrete-event simulation," in Proc. Int. Conf. Quality, Rel., Risk, Maintenance, Safety Eng., 2012, pp. 520-525.

[40] J. F. Lawless, Statistical Model and Methods for Lifetime Data, 2nd ed. Hoboken, NJ, USA: Wiley, 2011.

[41] R. B. Millar, Maximum Likelihood Estimation and Inference. 1st ed. New York, NY, USA: Wiley, 2011.

[42] O. F. Eker, F. Camci, A. Guclu, H. Yilboga, M. Sevkli, and S. Baskan, "A simple state-based prognostic model for railway turnout systems," IEEE Trans. Ind. Electron., vol. 58, no. 5, pp. 1718-1726, May 2011. 
[43] S. Sankararaman, M. Daigle, A. Saxena, and K. Goebel, "Analytical algorithms to quantify the uncertainty in remaining useful life prediction," in Proc. IEEE Aerosp. Conf., 2013, pp. 1-11.

[44] P. Wang, B. D. Youn, and C. Hu, "A generic probabilistic framework for structural health prognostics and uncertainty management," Mech. Syst. Signal Process., vol. 28, pp. 622-637, 2012.

[45] C. Louen, S. X. Ding, and C. Kandler, "A new framework for remaining useful life estimation using support vector machine classifier," in Proc. Control Fault-Tolerant Syst. Conf., 2013, pp. 228-233.

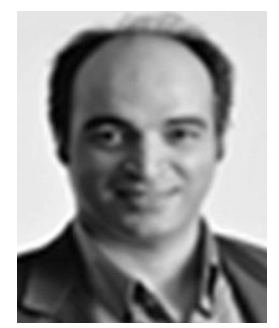

Alireza Alghassi (S'14) received the B.Sc. degree in electrical power engineering from Iran and Control System Engineering, Sheffield University, Sheffield, U.K., and the M.Sc. degree in power electronic engineering from Nottingham University, Nottingham, U.K. He is currently working toward the Ph.D. degree at the School of Applied Sciences, Integrated Vehicle Health Management Centre, Cranfield University, Cranfield, U.K.

$\mathrm{He}$ is currently a Researcher at Integrated VehiCranfield, U.K. cle Health Management Centre, Cranfield University,

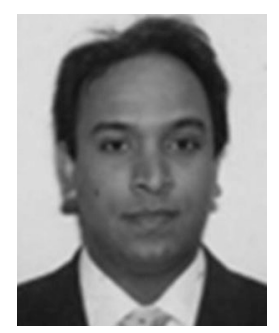

Suresh Perinpanayagam (M'13) graduated with the Master's of Engineering and Doctor of Philosophy degrees from Imperial College London, London, U.K.

$\mathrm{He}$ is currently a Lecturer at the Integrated Vehicle Health Management (IVHM) Centre, Cranfield University, Cranfield, U.K. He heads the Electronics Prognostics Group at the IVHM Centre, which works on highly accelerated life testing, electrothermal modeling, thermomechanical modeling, reliability and failure assessment capabilities for renewable energy systems, automotive, aerospace, and rail applications. This laboratory develops data-mining techniques for anomaly detection, diagnostics, prognostics, and predictive maintenance for power electronics from the above-mentioned industry sectors. His research group consists of one Research Fellow, five Ph.D. students, and Seven M.Sc. students.

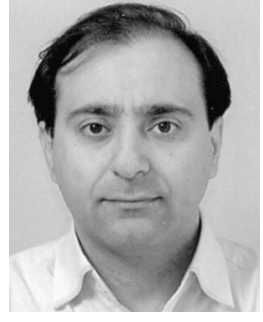

Mohammad Samie received the Ph.D. degree in advanced electronics from the University of the West of England (UWE), Bristol, U.K., in 2012.

$\mathrm{He}$ was involved in research on self-healing cellular architectures for biologically inspired highly reliable electronic systems funded by EPSRC, a joint research establishment between UWE and the University of Bristol. He is currently working as a Research Fellow for Integrated Vehicle Health Management, Cranfield University, Cranfield, U.K., which jointly funded by Boeing, Cranfield University, and number of other industrial partner to conduct research on system health management, power electronics, and self-healing systems.

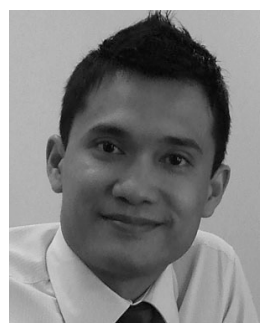

Tarapong Sreenuch received the B.Eng. degree in electronics and electrical engineering from the University of Surrey, Surrey, U.K., and the Ph.D. degree in control engineering from Cranfield University, Defence College of Management and Technology, Cranfield, U.K.

$\mathrm{He}$ is working on the Integrated Vehicle Health Management (IVHM) Centre, Cranfield, funded research projects related to software architecture and systems integration for IVHM systems. He has many years of experience in engineering software design and distributed systems. He is a Lead Developer at the IVHM Centre in design and development of IVHM related data process and software. He has more than 20 academic publications. He contributes to the teaching in M.Sc. in IVHM and M.Sc. in Autonomous Vehicle Dynamics and Control at Cranfield University. 
2014-10-02

\section{Computationally efficient, real-time, and embeddable prognostic techniques for power electronics}

Alghassi, Alireza

IEEE

Alghassi A, Perinpanayagam S, Samie M, Sreenuch T. (2015) Computationally efficient, real-time, and embeddable prognostic techniques for power electronics. IEEE Transactions on Power Electronics, Volume 30, Issue 5, May 2015, pp. 2623-2634

https://doi.org/10.1109/TPEL.2014.2360662

Downloaded from Cranfield Library Services E-Repository 